\title{
Nonlinear THF-FXLMS algorithm for active noise control with loudspeaker nonlinearity
}

\begin{abstract}
Adaptive algorithms are prevalently applied in the design of nonlinear active noise control (ANC) systems. The most important nonlinearity in ANC is the saturation effect produced by the electro-acoustical sensors and transducers. The dominant saturation nonlinearity is in the transducers, which can be represented by a Wiener model. An effective solution to mitigate such nonlinear distortion is to employ the Nonlinear Filtered-X Least Mean Square (NLFXLMS) algorithm. The controller compensates the nonlinearity using a model of the saturation effect represented by the scaled error function (SEF). However, the NLFXLMS is limited by two practical issues such that the degree of nonlinearity has to be known in advance and the SEF cannot be evaluated in real time. In this work, the NLFXLMS algorithm is modified by incorporating the tangential hyperbolic function (THF) to model the saturation effect of the loudspeaker. The proposed THF-NLFXLMS algorithm, models the nonlinear secondary path and applies the estimated degree of nonlinearity in the control algorithm design. The results show that the Wiener secondary path, with saturation nonlinearity represented by SEF, can be modelled by THF with a certain degree of accuracy and can yield a reasonable estimate of the degree of nonlinearity. The performance of the proposed algorithm is comparable with the benchmark NLFXLMS and is superior to the conventional FXLMS as well as the second order Volterra algorithm of similar computational complexity with the proposed algorithm.
\end{abstract}

Keyword: Active noise control; Secondary path modelling; Tangential hyperbolic function; THF-NLFXLMS; Wiener; SEF 